\title{
TU/e EmonOWEN

\section{8-GHz millimeter wave beam steered fiber wireless systems for 5G indoor coverage: architectures, devices, and links}

Citation for published version (APA):

Cao, Z., Zhao, X., Soares, F. M., Tessema, N. M., \& Koonen, A. M. J. (2017). 38-GHz millimeter wave beam steered fiber wireless systems for $5 \mathrm{G}$ indoor coverage: architectures, devices, and links. IEEE Journal of Quantum Electronics, 53(1), [7784730]. https://doi.org/10.1109/JQE.2016.2640221

\section{Document license: \\ TAVERNE}

DOI:

10.1109/JQE.2016.2640221

Document status and date:

Published: 01/02/2017

\section{Document Version:}

Publisher's PDF, also known as Version of Record (includes final page, issue and volume numbers)

\section{Please check the document version of this publication:}

- A submitted manuscript is the version of the article upon submission and before peer-review. There can be important differences between the submitted version and the official published version of record. People interested in the research are advised to contact the author for the final version of the publication, or visit the $\mathrm{DOI}$ to the publisher's website.

- The final author version and the galley proof are versions of the publication after peer review.

- The final published version features the final layout of the paper including the volume, issue and page numbers.

Link to publication

\section{General rights}

Copyright and moral rights for the publications made accessible in the public portal are retained by the authors and/or other copyright owners and it is a condition of accessing publications that users recognise and abide by the legal requirements associated with these rights.

- Users may download and print one copy of any publication from the public portal for the purpose of private study or research.

- You may not further distribute the material or use it for any profit-making activity or commercial gain

- You may freely distribute the URL identifying the publication in the public portal.

If the publication is distributed under the terms of Article 25fa of the Dutch Copyright Act, indicated by the "Taverne" license above, please follow below link for the End User Agreement:

www.tue.nl/taverne

Take down policy

If you believe that this document breaches copyright please contact us at:

openaccess@tue.nl

providing details and we will investigate your claim. 


\title{
38-GHz Millimeter Wave Beam Steered Fiber Wireless Systems for 5G Indoor Coverage: Architectures, Devices, and Links
}

\author{
Z. Cao, Member, IEEE, X. Zhao, F. M. Soares, \\ N. Tessema, and A. M. J. Koonen
}

\begin{abstract}
Millimeter wave (mm-wave) beam steering is a key technique for the next generation (5G) wireless communication. The 28 and $38-\mathrm{GHz}$ bands are widely considered as the candidates for 5G. In the context of indoor coverage, fiberwireless systems with multiple simplified remote antenna sites are attractive to avoid the indoor coverage problem caused by the high wall penetration loss of $\mathrm{mm}$-wave signals. To allow enough antenna gain at the $\mathrm{mm}$-wave bands, radio beam steering (and beamforming) is desired. Combining fiber-wireless system with remotely controlled photonic mm-wave beam steering can bring significant advances in terms of energy efficiency and cost. In this paper, we explore two kinds of indoor fiber-wireless network architectures for such mm-wave beam steering. Then, we discuss and investigate the key enabling device, which is an arrayed waveguide grating feedback loop (AWG-loop). Based on the AWG-loop, we further design two fiber-wireless links to accommodate the two network architectures. Both links with bit rates from $50 \mathrm{Mb} / \mathrm{s}$ to $8 \mathrm{~Gb} / \mathrm{s}$ per spatial channel are experimentally demonstrated with a $38-\mathrm{GHz}$ carrier frequency. The advanced reversely modulated optical transmitter and half-cycled 16 quadrature amplitude modulation (QAM-16) are employed to realize a simplified mm-wave beam steered fiber-wireless link with the record-breaking $16-\mathrm{b} / \mathrm{s} / \mathrm{Hz}(4$ spatial channels $\times 4$ bits/s/Hz) spatial-spectral efficiency in its kind.
\end{abstract}

Index Terms-5G, mm-wave, optical mm-wave beam steering, photonic integrated circuit, optical tunable delay line.

\section{INTRODUCTION}

$\mathbf{T}$ HE current explosion of communication traffic is driven by an insatiable appetite for high speed internet connectivity and video-based content delivery to wireless and mobile terminal users, especially for indoor scenarios. The next generation wireless networks (5G) target to provide 1000 times higher wireless area capacity in 2020 compared

Manuscript received August 9, 2016; revised October 26, 2016; accepted November 22, 2016. Date of publication December 15, 2016; date of current version January 11, 2017. This work was supported in part by the European Research Council within the FP-7 Program under the Grant 291632 (BROWSE) and Grant 257210 (PARADIGM) and in part by the Open Fund of IPOC (BUPT).

Z. Cao, X. Zhao, N. Tessema, and Ton Koonen are with the Department of Electrical Engineering, COBRA Research Institute, Eindhoven University of Technology, NL 5600 MB Eindhoven, The Netherlands (e-mail: z.cao@tue.nl; x.zhao.1@tue.nl; n.tessema@tue.nl; a.m.j.koonen@tue.nl).

F. M. Soares is with the Fraunhofer Institute for Telecommunications, Heinrich Hertz Institute, 10587 Berlin, Germany (e-mail: francis-co.soares@hhi.fraunhofer.de).

Color versions of one or more of the figures in this paper are available online at http://ieeexplore.ieee.org.

Digital Object Identifier 10.1109/JQE.2016.2640221 to the one in 2010 [1]. To boost the capacity in the spectral domain, the millimeter wave (mm-wave) communication is a promising solution by exploring the huge bandwidth at higher frequency [2], [3]. There are two challenges for mm-wave communications: one is the high penetration loss through walls, which makes inter-room connection/coverage difficult; the other is the small electrical aperture induced low antenna gain. Fiber-wireless systems with loss-less fiber links and multiple simplified remote antenna sites can overcome the inter-room connection/coverage problem, while the issue of low antenna gain can be overcome by beam steering (and beamforming) techniques. Samsung recently proposed and demonstrated a mm-wave communication empowered by beam steering [3], which is based on electronic devices. For broadband beam steering, true time delay (TTD) is required. However, the electronic integrated circuits (for instance (Bi) CMOS) suffer from high loss at high frequencies. Optical true time delay (OTTD) with inherent low loss and broad bandwidth can be used for broadband $\mathrm{mm}$-wave beam steering to avoid the beam squint problems [4]-[13]. Fiber-wireless systems incorporating optical true time delay radio beam steering have been proposed for indoor networks [5], [6]. In such work, OTTD is beneficial to the radio over fiber (RoF) systems since no additional electrical-optical and optical-electrical conversion is required. However, the optical tunable delay lines (OTDLs) employed in [5], [6] are based on bulk-optics components, which limit their further applications. The integrated OTDLs are of demand [14]-[17] for compact and stable optical mm-wave beam steering. Recently, we designed and characterized a remotely tunable integrated OTDL based on an arrayed waveguide grating feedback loop (AWG-loop). Since the AWG is used as both the wavelength multiplexer (MUX) and the de-multiplexer (de-MUX), the AWG-loop allows a compact, fabrication tolerant and scalable solution for integrated OTDL [18]. Thanks to this AWG-loop, a series of advanced system features are guaranteed: 1) the antenna sites can be simplified with a single remotely tunable OTDL chip without active control; 2) multiple beams supporting multiple users can be realized simultaneously due to its linear feature; 3 ) it is low cost, compact and power efficient compared with its discrete component counterpart.

Enabled by our novel integrated AWG-loop, we propose and experimentally demonstrate two novel optical $\mathrm{mm}$-wave beam steered fiber-wireless systems at $38 \mathrm{GHz}$ for $5 \mathrm{G}$ indoor 


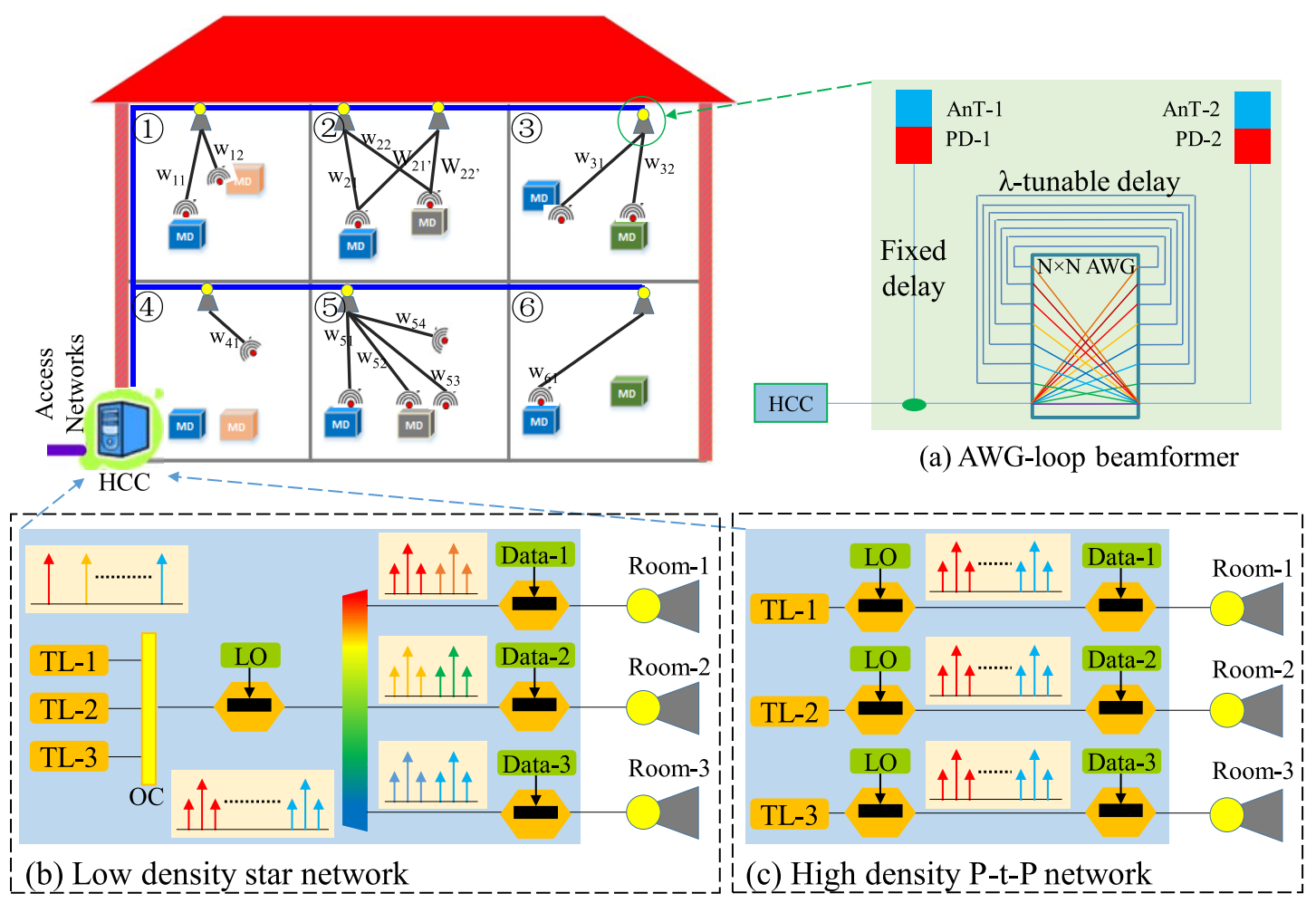

Fig. 1. The principle of AWG-loop based integrated optical tunable delay line (OTDL), (a) AWG-loop delay network; (b) delay at Out-2 of (a), (c) delay at Out-1 of (a). OS: optical power splitter; Signal: optical mm-wave signal; CODL: compensation optical delay line.

coverage, one for low density star architecture, the other for high density point-to-point (P-t-P) architecture. Two fiberwireless links are designed and experimentally investigated for such two architectures. In these links, the fiber-wireless data transferring with bit rates from $50 \mathrm{Mb} / \mathrm{s}$ to $8 \mathrm{~Gb} / \mathrm{s}$ are demonstrated with mm-wave beam steered by remote wavelength tuning. The whole paper is organized as follows. In Section II, two indoor beam steered fiber-wireless network architectures are proposed and discussed. The properties and operation principle of the proposed integrated AWG-loop are explored in Section III. In Section IV and Section V, the beam steered fiber-wireless links designed for low-density star architecture and high-density P-t-P architecture are discussed and experimentally studied. Finally, the whole paper is concluded in Section IV. This paper is an extension of our work presented in OFC2016 [19].

\section{ARChitecture OF Indoor mm-WaVe BeAm STEERED Fiber WiRELESS SYSTEM}

Fig. 1 shows a mm-wave beam steered fiber-wireless system in an indoor scenario. The wireless services delivered by an external access network are processed via a home control center (HCC), which serves as a gateway. After the HCC, the processed wireless signals are modulated on optical carriers and then distributed via an indoor fiber-wireless network. In each room, photonic mm-wave beamformers based on AWG-loops, as shown in Fig. 1(a), are installed at the end of the network as remote access points. The wireless signal carried on optical carrier is split into two branches, one directly to a photodiode (PD-1) and its cascaded antenna, the other to the other photodiode (PD-2) through an AWG-loop. The AWG-loop is, in essence, a step-wise wavelength-tunable discrete delay unit. It is elaborated in Section III. When the wavelength of an optical carrier is tuned, the delay at PD-1 is fixed while the one at PD-2 is step-wisely tuned. It tunes the delay difference between the two antennas, which results in the mm-wave beam steering. The steps of tuned beams in an AWG-loop depend on the number of tunable wavelengths. To avoid cross talk, there is a minimal value for the spectral spacing between each wavelength. Thus, the spectral range of the incoming signal defines the upper boundary of the beam density. Here we propose two network architectures: one is the star architecture for low density of tuned beams; the other is the P-t-P architecture for high density of tuned beams. The low density architecture is designed for low capacity systems with low complexity while the high density one aims for high capacity systems.

As shown in Fig. 1(b), all the wavelengths generated from tunable lasers (TL-1/2/3) in the start architecture are combined with the spectrum schematically shown in inset $i$. Different colors denote different wavelengths. The combined wavelengths are then modulated by a local oscillator (LO) via an up-conversion modulator (UpCon-Mod) with spectrum shown in inset ii. Afterwards, the wavelengths are separated into a series of wavelength groups by a wavelength de-MUX for different rooms. The spectra after de-MUX are shown in inset iii/iv/v. The services provided for each room are modulated on each wavelength group. The beam density in a room is determined by the wavelength tuning range of its allocated wavelength group. Since the whole spectral range is 
(a) AWG-loop delay network

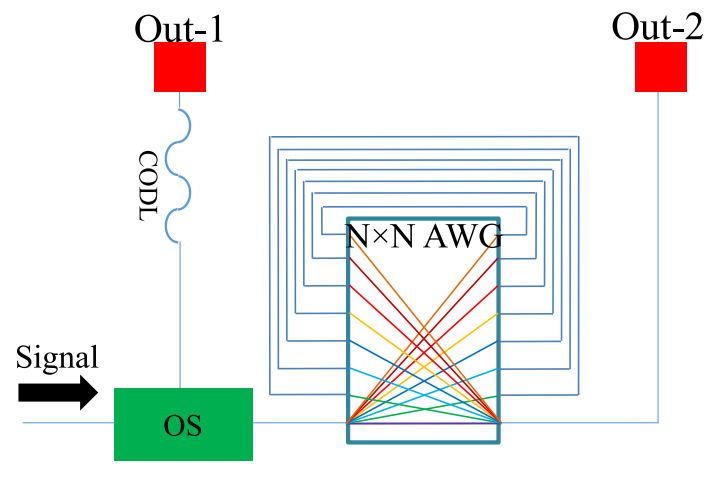

(b) Delay at Out-2
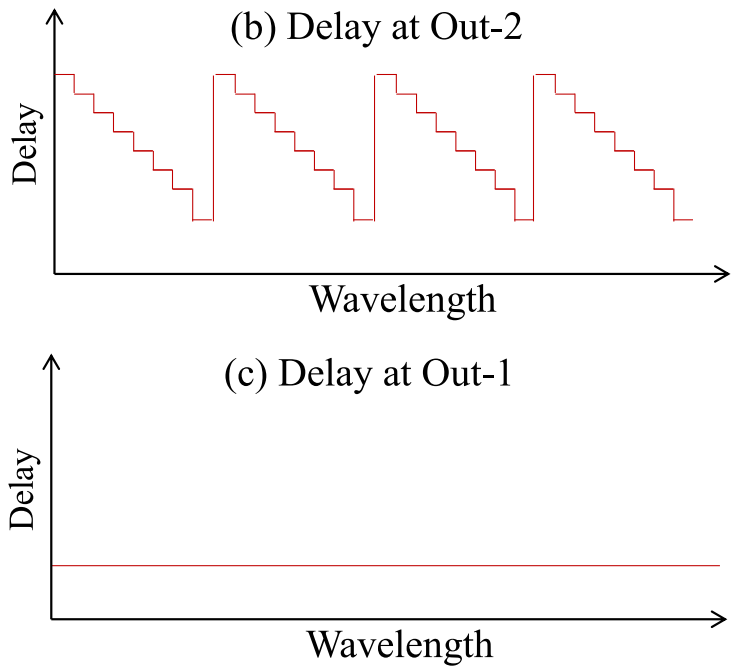

Fig. 2. The principle of AWG-loop based integrated optical tunable delay line (OTDL), (a) AWG-loop delay network; (b) delay at Out-2 of (a), (c) delay at Out-1 of (a). OS: optical power splitter; Signal: optical mm-wave signal; CODL: compensation optical delay line.

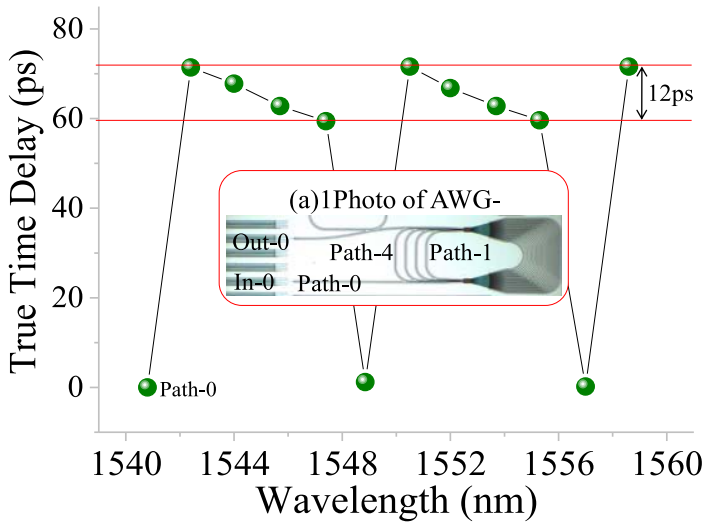

Fig. 3. The measured delays of different delay channels of the AWG-loop.

split into many pieces for different rooms, the beam density of each room is low.

As shown in Fig. 1(c), the high density P-t-P architecture provides separate fiber channels for each room. It means that the whole spectral range can be fully used for each room, as shown in inset $\mathrm{i} / \mathrm{ii} / \mathrm{iii}$, resulting in a high beam density. However, for each room, separate up-conversion modulators are on demand. It is clear that the advantage of the high density one is achieved with the sacrifice of complexity and cost.

\section{Integrated Remotely-Tunable Delay Line}

The proposed integrated AWG-loop and its corresponding AWG-loop delay network (ALDN) to serve as a photonic mm-wave beamformers are shown in Fig. 2. An experimental system based on such ALDN is shown in Fig. 5, which will be detailed in the following part. The 1-by-2 ALDN shown in Fig. 2(a) includes an optical power splitter (OS) and an AWG-loop. The optical signal is split into two paths: one directly goes to the first output (Out-1) and the other goes through an AWG-loop to the second output (Out-2). As shown in Fig. 2(a), the AWG-loop is formed by the

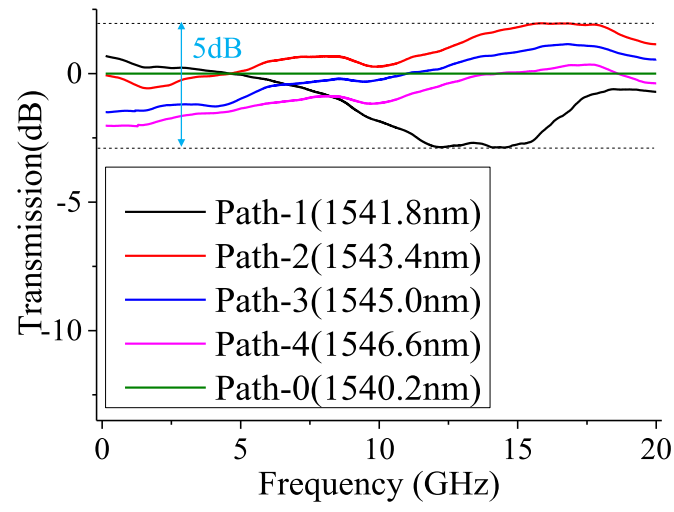

Fig. 4. The measured RF transmission of different paths of the AWG-loop.

N-by-N spectral-cyclic AWG, which is employed as both the wavelength MUXand the de-MUX. The AWG is cyclic in optical spectrum, which allows further interleaved operation for 2-D optical mm-wave beam steering [4]. The feedback loops (optical waveguides) connect $\mathrm{N}-1$ pairs of inputs and outputs of the AWG in a symmetric configuration for recirculating operations and one pair of input and output is left as the input and output of the AWG-loop. This AWG-loop is topologically equivalent to two AWGs in series with a delay element in between, which works as a stepwise OTDL. As shown in Fig. 2(b), the delay of Out-2 can be selected by tuning the wavelength of the signal. The delay of Out- 1 is shown in Fig. 2(c). A compensation optical delay line (CODL) is employed to compensate the travel time of the AWG-loop to avoid initial delay offset between Out-1/-2. By re-using the same AWG as MUX and de-MUX, its footprint can be significantly reduced (approximately by half) and the relative spectral mis-alignment between the MUX and de-MUX does not exist anymore. More details regarding such ALDN can be found in [4]. Such an ALDN also provides advanced system features: 1) the antenna sites can be simplified with a single remotely tunable OTDL chip without active control; 2) multiple beams supporting multiple users can be realized simultaneously due 


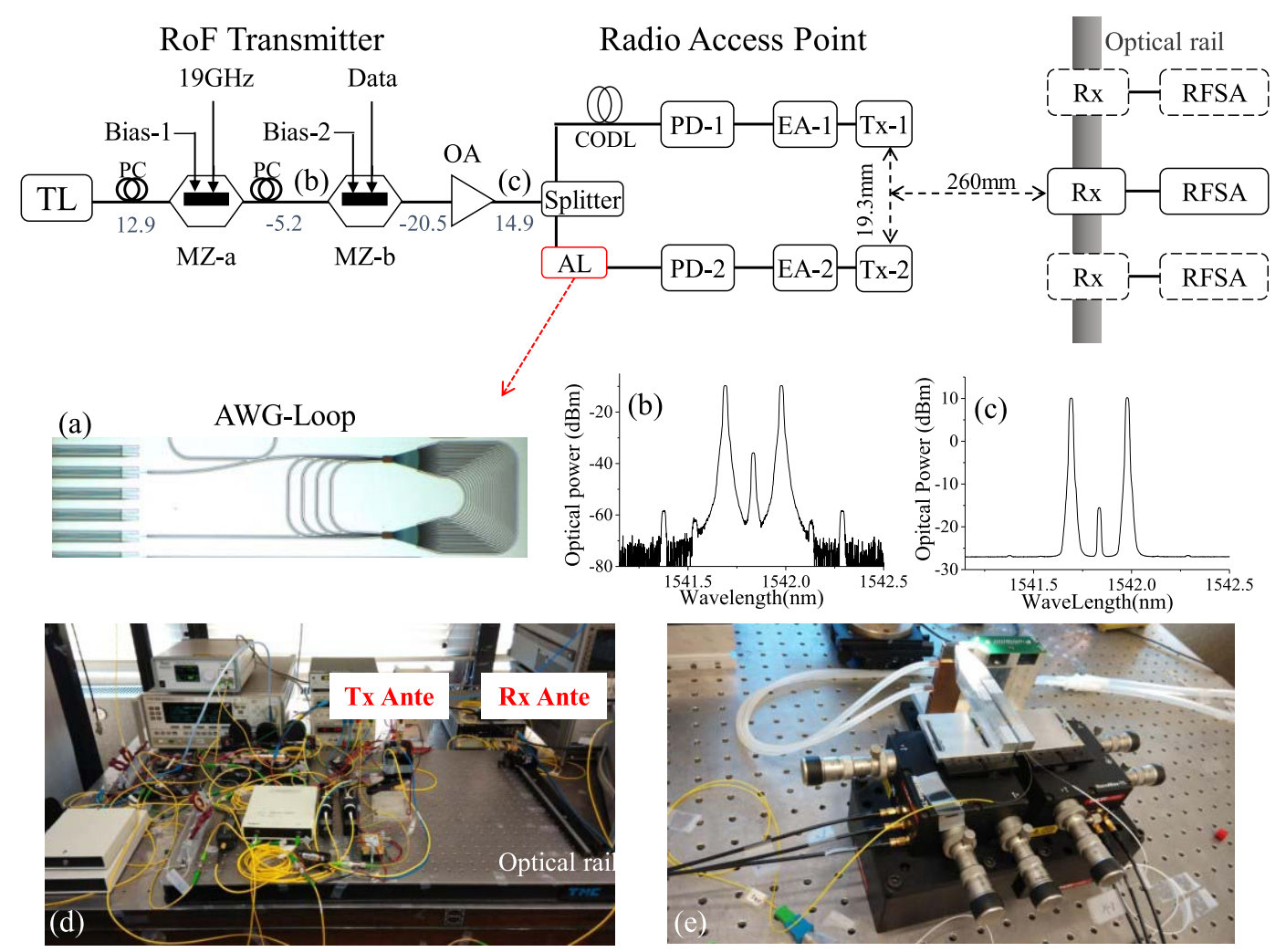

Fig. 5. The experimental setup of 38-GHz mm-wave beam-steered RoF system for star architecture, (a) the photo of the AWG-loop chip; (b)-(c) the measured optical spectrum; (d) the photo of the beam-steered RoF system with antenna subsystem; (e) the photo of fiber coupling subsystem.

to its linear feature; 3) it is low cost, compact and power efficient compared with its discrete component counterpart.

The photo of the fabricated integrated AWG-loop is shown in Fig. 3(a). The core component of the AWG-loop is a spectral-cyclic 5-by-5 AWG. Its grating central wavelength $\lambda_{\mathrm{c}}$ is designed at $1550 \mathrm{~nm}$ and the grating order is designed to be 174. The free spectral range (FSR) is $8 \mathrm{~nm}(1000 \mathrm{GHz})$. The channel spacing (CS) is $1.6 \mathrm{~nm}(200 \mathrm{GHz})$ and its $-3-\mathrm{dB}$ passband is $0.52 \mathrm{~nm}(65 \mathrm{GHz})$. The waveguides connect four pairs of input and output to form the feedback loops with different delays. One pair of input and output of the AWG (In-0, Out-0) is used as the input and output of the AWG-loop and is connected to two spot size convertors (SSCs). Deep-etched waveguides are used for all these connections. The footprint of the AWG-loop is $2.6 \mathrm{~mm} \times 1.2 \mathrm{~mm}$. The maximum insertion loss of the AWG-loop is $6.5 \mathrm{~dB}$ without SSC coupling loss. With gain section on such platform, this loss can be easily compensated. The maximum delay (Path-4) is designed to be $12.5 \mathrm{ps}$ for $\pi$ phase shift at $40 \mathrm{GHz}$. The designed delays of Path-1 to Path-4 increase progressively with a range of 12.5 ps. A time domain correlation method is used for delay measurement. As shown in Fig. 3, the measured delays linearly increases from Path-1 to Path-4 with a delay range of $12 \mathrm{ps,}$ which is a little smaller than the one we expected. The differences between the designed delays and the measured ones for Path- 1 to Path- 4 are $-0.5,0.1,-0.76$ and 0 ps from 1540 to $1548 \mathrm{~nm}$. The acceptable match between the designed delays and measured ones demonstrate a high design accuracy. As shown in Fig. 4, we measured the radio frequency (RF) transmission curve of the AWG-loop by an electrical vector network analyzer (VNA) with a measurement range from $130 \mathrm{MHz}$ to $20 \mathrm{GHz}$. The Path-0 without feedback loop is used as a referenced path with optimized alignment of optical carrier and the passband center of the employed AWG channel. The -3-dB bandwidth of the AWG is $65 \mathrm{GHz}$, which is much higher than the frequency range of the VNA. Therefore the AWG filtering effect does not contribute significantly to the RF bandwidth while the limited bandwidth of the employed optical transmitter and receiver does. All measured results are then calibrated by the measured transmission of Path- 0 to remove the disturbance from the optical transmitter and receiver. The results suggest that the transmission fluctuation is less than $5 \mathrm{~dB}$. The phase-frequency can be found in [20], which demonstrates its broadband true time delay feature.

\section{FibER-WireleSS LINK FOR LOW DENSITY STAR ARCHITECTURE}

The mm-wave beam steered fiber-wireless link for star architecture is schematically shown in Fig. 5. The datacarrying optical mm-wave is generated by the radio-over-fiber (RoF) transmitter and then delivered to the radio access point. The tuning of the optical carrier wavelength results in a change of the differential delay between the two photodiodes and finally makes the mm-wave beam steered spatially. The proofof-concept experimental setup is described in the following. The 12.9-dBm optical carrier from a tunable laser (TL) passes to a Mach-Zehnder modulator (MZM, MZ-a shown in Fig. 5) via a polarization controller (PC). The $19-\mathrm{GHz}$ clock signal 
is applied to MZ-a biased at its null point for optical carrier suppression. In this way, the $38-\mathrm{GHz}$ carrier frequency can be generated after optical-electrical conversion. The generated $-5.2-\mathrm{dBm}$ optical $\mathrm{mm}$-wave is then modulated by the second MZM (MZ-b) after a PC. The 25-MSymbol/s QPSK signal at $500-\mathrm{MHz}$ intermediate frequency generated from a vector signal generator (R\&S SMU200A) is applied on MZ-b. The data rate is limited by the vector signal generator. In principle, the integrated ALDN subsystem provides a bandwidth limitation as large as $65 \mathrm{GHz}$ here. The resulted $-20.5-\mathrm{dBm}$ optical signal is then amplified to $14.9 \mathrm{dBm}$ before 50 -m single mode fiber delivery. The optical spectra after MZ-a and MZ-b are shown respectively in Fig. 5(b) and (c). The optical signal arrives at the radio access point and is split into two paths. One directly connects to a photodiode (PD-1) with a discrete tunable delay line to compensate the delay offset between the two paths. The launched optical power of PD-1 is $8.7 \mathrm{dBm}$. The other passes through the integrated AWG-loop by two cleaved single mode fibers for coupling. The photo of the fiber coupling system is shown in Fig. 5(e). The output signal with $2.4 \mathrm{dBm}$ power is amplified to $10.3 \mathrm{dBm}$ and then launched into a photodiode (PD-2) for optical-electrical conversion. The converted signals are then amplified by two $40-\mathrm{GHz}$ band amplifiers (EA-1 and EA-2). The outputs of EA-1/2 are separately connected to two identical $38-\mathrm{GHz}$ aperture antennas $(\mathrm{Tx}-1$ and $\mathrm{Tx}-2)$ to transmit the data signals wirelessly. The center-to-center distance $d$ between Tx-1/-2 is $1.93 \mathrm{~cm}$. To receive the wireless signals, a $38-\mathrm{GHz}$ aperture antenna $(\mathrm{Rx})$ is mounted on an optical rail at a forward distance of $260 \mathrm{~mm}$. The Rx antenna can be moved along the rail. The measurement is carried out with different lateral distances along the optical rail. A photo of the whole fiber-wireless system including the antennas is shown in Fig. 5(d). The received 38-GHz signal is then analyzed by a radio frequency spectral analyzer (RFSA) to obtain the received power, phase noise and error vector magnitude $(\mathrm{EVM})$ of the QPSK signal.

The measured phase noise of the $38-\mathrm{GHz}$ signal is shown in Fig. 6. It indicates that the phase noise performance can be improved if the optical signal goes through the integrated AWG-loop). Since the out-of-band noise is partially filtered out due to the passband effect of the AWG-loop, the conversion from additional noise to phase noise is relieved, which reduces the corresponding phase noise. We measured the received power of the $38-\mathrm{GHz}$ signal along the optical rail as shown in Fig. 5(d) to obtain the mm-wave beam profile and to observe the mm-wave beam steering. The center of the two transmitter antennas (Tx-1/-2) and the origin of the optical rail are aligned. The measured received power versus the offset of the optical rail origin (referred as ' $\mathrm{X}$-axis offset') is shown in Fig. 7 with its simulation counterpart. The simulation is performed based on the basic expression of the array factor shown below:

$$
A F(\theta)=\sum_{n=1}^{N} I_{n} \exp (-j \beta n d \sin \theta)
$$

The assumption behind Eq. (1) is that the forward distance $(260 \mathrm{~mm})$ is much longer than the element antenna distance

\section{Phase noise comparison}

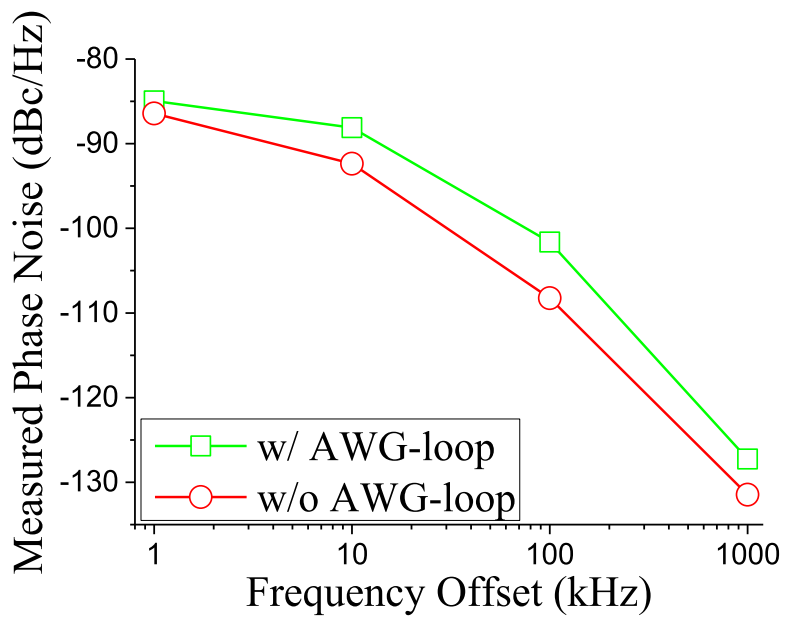

Fig. 6. The measured phase noise of different delay channels of the AWG-loop.

$d(19.3 \mathrm{~mm})$, which results in the same arrival angle $(\theta)$ for $\mathrm{Tx}-1$ and $\mathrm{Tx}-2$. The additional phase compensation is added since the forward distance from the transmitter antennas $(\mathrm{Tx}-1 /-2)$ is not that long. The engaged aperture antennas can be considered as semi-omni-directional antennas. Their far-field patterns cover the whole hemisphere with a small deviation. Thus we do not take the far-field pattern of the aperture antennas into account in our simulation. In the simulation, the amplitude coefficients of the two antennas are set to 1 and 0.675 respectively corresponding to the optical powers launched to the photodiodes. The phase shifts of the two antennas in the simulation are converted from the measured delays of the integrated AWG-loop. The peak of the simulated power curve is then normalized to the peak of the experimental results for all cases.

Fig. 7(a) and (b) are based on the same data but with different presentations for illustration convenience. As shown in Fig. 7(b), the simulated and experimental results are depicted one by one for all four wavelengths (delays). We can clearly see that the experimental results match well with the simulated ones in terms of peak/null locations and their periodicities. The difference between the two results may be introduced by the variation of the AWG-loop and the inevitable mm-wave reflection in the real environment.

In Fig. 7(a), the simulated and experimental results are separated to allow better illustration of the trends. The different wavelengths can select different paths (delays) of the integrated AWG-loop, which results in the different beam directions. As the wavelength increases from $1541.8 \mathrm{~nm}$ to $1546.6 \mathrm{~nm}$, the delay decreases from 12 ps to 0 ps as shown in Fig. 3, which causes the beam peaks to move from the left side to the right side. There are more than one peaks shown in the beam profiles for all delay (wavelength) cases. For phased array antennas, it is well known that the grating lobes can be suppressed if the element antenna distance $d$ is smaller than one half of the mm-wave wavelength $\left(\lambda_{\mathrm{mm}}\right)$. In our experiment, due to the bulk aperture antennas, the 

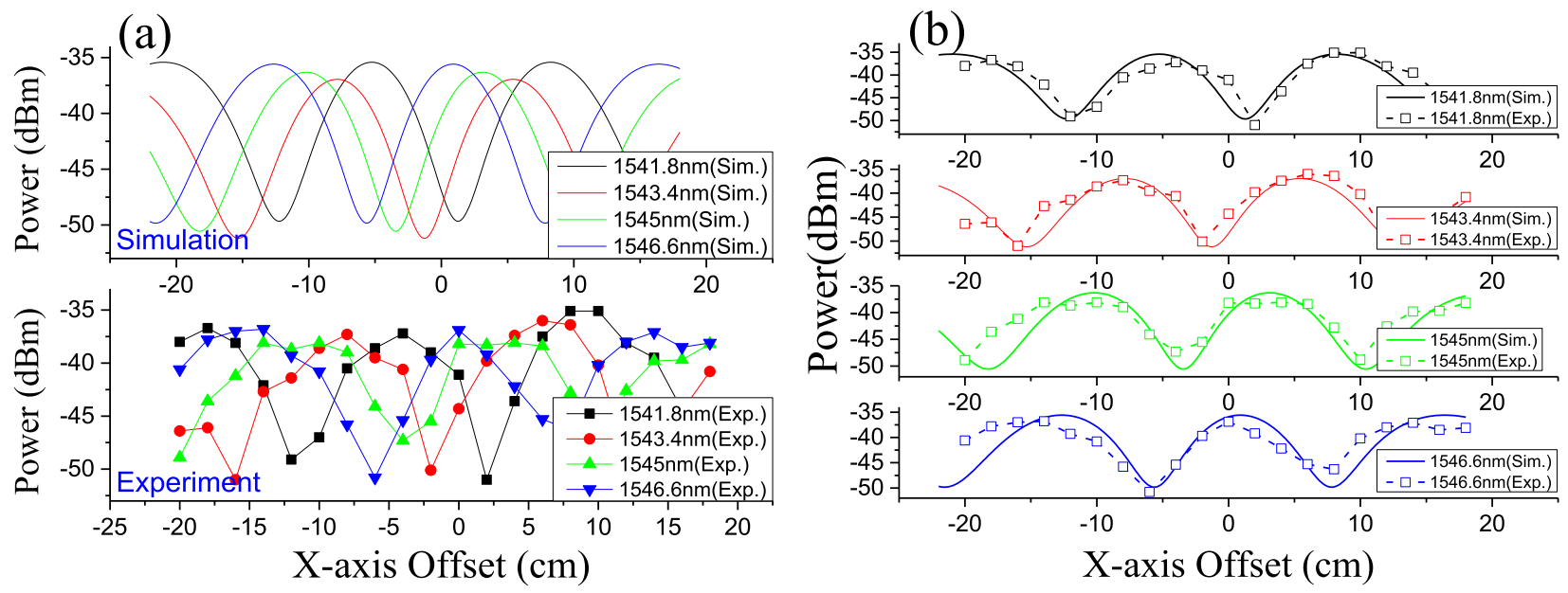

Fig. 7. The received power versus $x$-axis offset for simulation and experiment, (a) the separated figures for the simulated and the experimental results; (b) the one-by-one comparison between the simulated results and experimental ones for each delay.

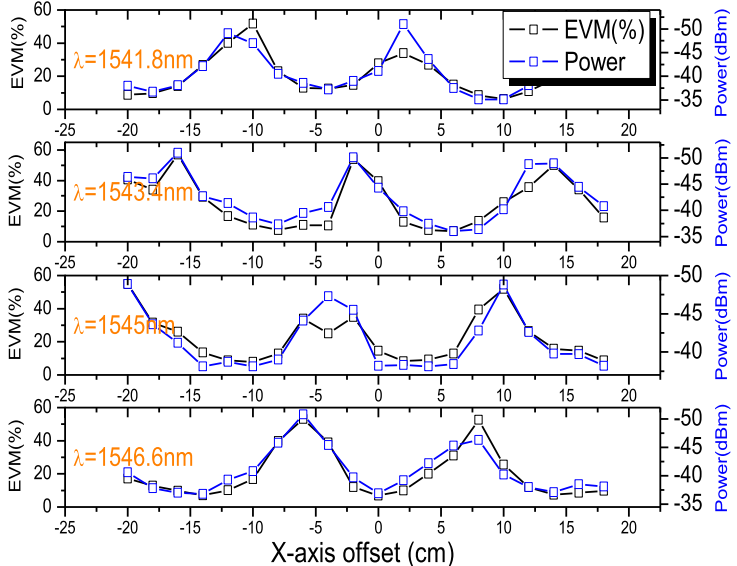

Fig. 8. The measured error vector magnitude and power versus $\mathrm{x}$-axis offset for the received $38-\mathrm{GHz}$ signal.

distance $d$ is $1.93 \mathrm{~cm}$, which is much larger than one half of $\lambda_{\mathrm{mm}}(0.39 \mathrm{~cm})$, thus the grating lobes exist and result in more than one peaks in the beam profiles. The widely used patch antenna with smaller size can solve such problem. The power suppression ratios for all delays are more than $14 \mathrm{~dB}$. This can be further improved if the power balance of PD-1/-2 can be conserved. The simulation well predicts such imbalance as shown in Fig. 7(a). The experimental results show that the beam peaks of different delays are with slight differences. This may be induced by the variation of insertion loss of the AWG-loop and the mechanism twist induced connection loosing.

The measured EVM of the QPSK signal on $38-\mathrm{GHz} \mathrm{mm}-$ wave is shown in Fig. 8 with the corresponding received power. The two curves are normalized to allow better visualization. The corresponding constellations are shown in Fig. 9. The EVM curves agree well with the power curves, indicating that the beam steering induced power variance determines the quality of the received QPSK signal. When the mm-wave beams direct to the receiving antenna, the EVMs can be smaller than $9 \%$, and the constellations are well converged as shown in the first line of Fig. 9. As shown in Fig. 7, around 14-dB power difference between the beam and the

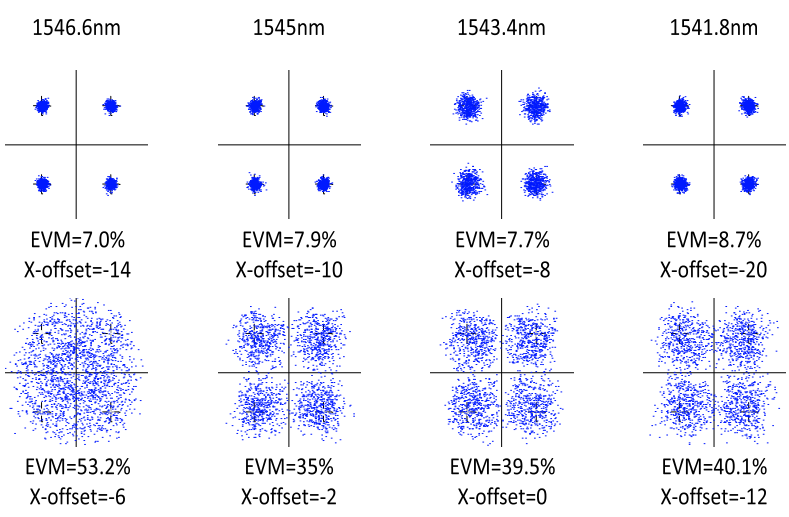

Unit of X-offset is $\mathrm{cm}$

Fig. 9. The measured constellations for received QPSK signal on $38-\mathrm{GHz}$ carrier frequency.

null can be achieved. This means for the nulls, the received power is reduced by $14 \mathrm{~dB}$. Such low received power even cannot allow the demodulation of the QPSK signal. As shown in Fig. 9, the constellation in the 1546.6-nm case with $-6-\mathrm{cm}$ $\mathrm{x}$-axis offset demonstrates an unsuccessful demodulation of the QPSK signal. The EVM can be reduced from around $40 \%$ to around $7 \%$, indicating around 6 times quality enhancement. The 14-dB spatial power focusing indicates that the power leaked from one channel to the other neighbor spatial channels can be significantly reduced by $14 \mathrm{~dB}$, which can relieve the heavy task of digital signal processing.

\section{Fiber-Wireless LinK FOR High Density POINT-TO-Piont ARCHITECTURE}

As discussed in Section II, the high density architecture aims for high capacity systems but with high complexity. Here we design and investigate the $\mathrm{mm}$-wave beam steered fiber -wireless link based on the same photonic mm-wave beamformer with Section IV. To reduce the modulators used in optical transmitters, a single reversely modulated dualelectrode MZM without electrical mixers is used for high optical modulation efficiency and electrical-bottleneck-free 


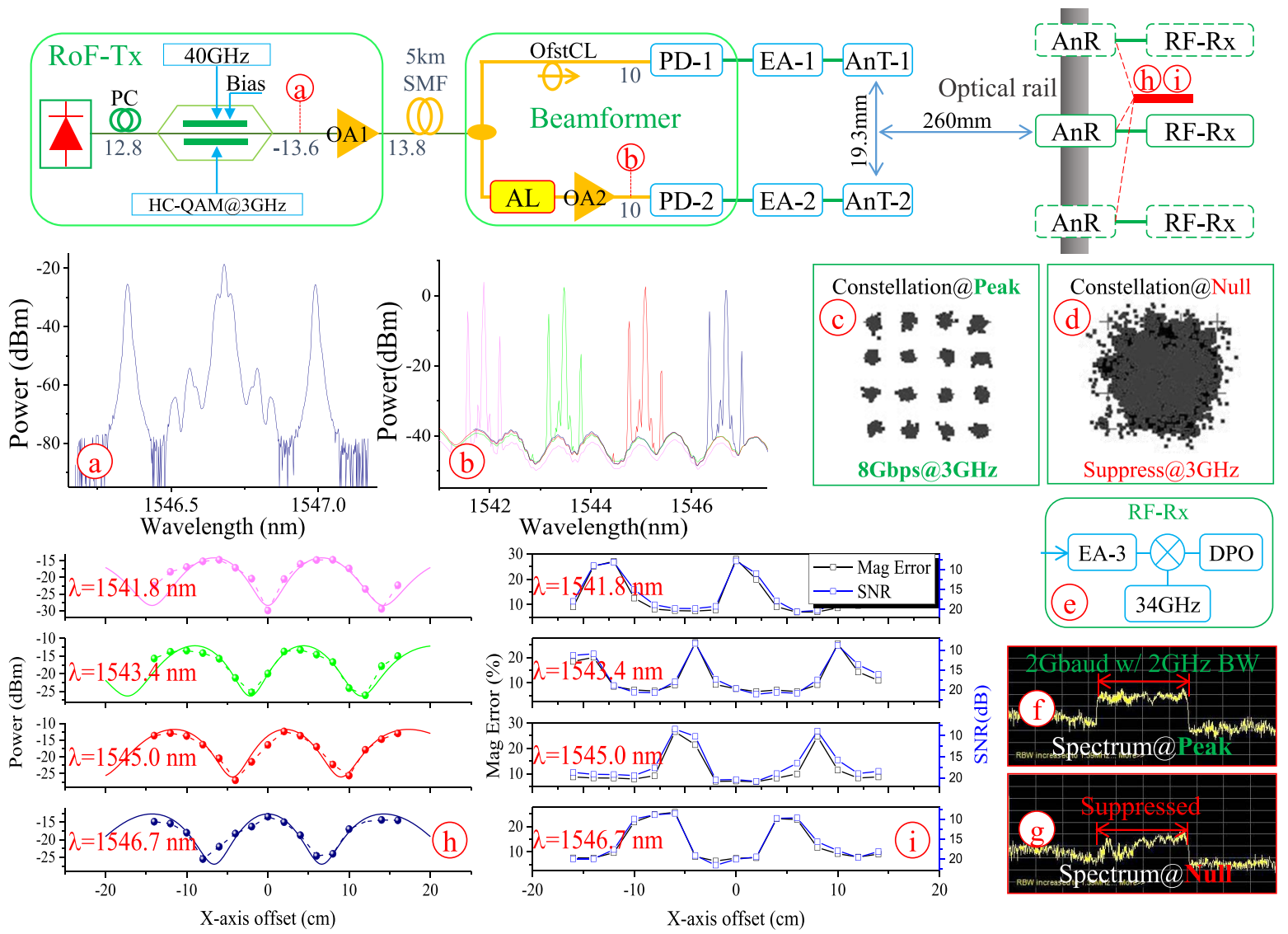

Fig. 10. Experimental setup and results of simplified fiber-wireless link for high density point-to-point architecture.

operation. In order to boost the system capacity and performance, the single carrier half-cycled (HC) QAM-16 modulation format is employed. It keeps the same spectral efficiency as OFDM but enables a lower peak-to-average ratio compared to OFDM. With these enabling techniques, a simplified fiberwireless link with beam steering capability is demonstrated to exceed 16 bits $/ \mathrm{s} / \mathrm{Hz}$ (4 spatial channels $\times 4$ bits $/ \mathrm{s} / \mathrm{Hz}$ ) spatialspectral efficiency. The bit rate of a spatial channel is $8 \mathrm{~Gb} / \mathrm{s}$ and the channel isolation is $14 \mathrm{~dB}$.

The reversely modulated DE-MZM mm-wave RoF link based on the integrated AWG-loop is schematically shown in Fig. 10. The $12.8 \mathrm{dBm}$ wavelength-tunable optical carrier passes to a $10 \mathrm{GHz}$ DE-MZM via a polarization controller. A $40 \mathrm{GHz}$ clock signal is applied to the upper arm, and an 8 Gb/s HC QAM-16 signal (2 Gbaud with $2 \mathrm{GHz}$ bandwidth, raised cosine shaping, $\beta=0.01$, spectrum shown in Fig. 10(f)) at $3 \mathrm{GHz}$ is applied to the lower arm. Here we use a cheap low-bandwidth DE-MZM as the HC QAM-16 signal is modulated in the low frequency range $(<4.5 \mathrm{GHz})$. The limited-bandwidth induced power fading just decreases the $40 \mathrm{GHz}$ LO power rather than the HC QAM-16 signal quality. The MZM is biased at its power null point (E-field linear point) to allow high modulation efficiency. The data is modulated close to the center while the LO is modulated as sidebands with a so-called reversely modulated manner. The spectrum of the modulated optical signal is shown in Fig. 10(a). Less than $10 \mathrm{~dB}$ carrier-to-sideband suppression is achieved for high modulation efficiency. After $5 \mathrm{~km} \mathrm{SMF,} \mathrm{the} \mathrm{optical}$ signal is split into two paths. One directly connects to a photodiode (PD-1) with a bulk tunable offset compensation line (OfstCL). The other passes through the AWG-loop coupled by two cleaved SMFs. The features of AWG-loop are already discussed in Section III. The converted signals are then amplified by two $40 \mathrm{GHz}$ band amplifiers (EA-1 and EA-2) to transmit the HC QAM-16 signal wirelessly via two identical $40 \mathrm{GHz}$ aperture antennas (AnT-1/-2). A $40 \mathrm{GHz}$ aperture antenna $(\mathrm{AnR})$ for receiving the wireless signal is moved along an optical rail. A RF receiver (RF-Rx) (shown in Fig. 10(e)) comprises an electrical down-converter and a real time 50 GSaps oscilloscope (DPO) for signal demodulation and analysis. Geometric parameters for the antenna locations can be found in Fig. 10. Four spatial channels can be steered by tuning the wavelength of the optical carrier. The spectra of the tuned wavelengths at 1541.8, 1543.4, 1545.0, $1546.6 \mathrm{~nm}$ are depicted in Fig. 10(b). Their intentional asymmetric profiles form single sideband modulation, which can avoid frequency fading effects.

As shown in Fig. 10(h), the measured power (dot) along $\mathrm{X}$-axis and its simulated counterpart (line) are depicted one by one for all four wavelengths (delays). We can clearly see that the experimental results match well with the simulated ones in terms of peak/null locations and their periodicities. In the 
real environment, the difference between two results may be introduced by the inevitable mm-wave reflection. Wavelength tuning results in delay tuning and further controls radio beam directions. As the wavelength increases from $1541.8 \mathrm{~nm}$ to $1546.6 \mathrm{~nm}$, the delays decreases from $12 \mathrm{ps}$ to $0 \mathrm{ps}$ as shown in Fig. 3, which results in the beam peaks moving from the left side to the right side. For phased array antennas, it is well known that the side lobes can be suppressed if the element antenna distance $d$ is smaller than one half of the mm-wave wavelength $\left(\lambda_{\mathrm{mm}}\right)$. In our experiment, due to the bulk aperture antennas, the distance $d$ is $1.93 \mathrm{~cm}$, which is much larger than one half of $\lambda_{m m}(0.39 \mathrm{~cm})$, thus the side lobes exist, resulting in more than one peak in the beam profiles as shown in Fig. 10(h) and (i). They can be easily avoided by using patch antennas. The power suppression ratios for all delays are more than $14 \mathrm{~dB}$. This means a $14 \mathrm{~dB}$ channel isolation, which is good enough for independent spatial channels. It can be further improved with a good power balance between PD-1/-2 or with a larger antenna array. The simulation well predicts such unbalance as shown in Fig. 10(h). The signal-to-noise ratio (SNR) shown in Fig. 10(i) is measured based on the received power of wireless signals, which exhibits a good matching with the measured power in Fig. 10(h). The measured EVM of the $8 \mathrm{~Gb} / \mathrm{s}$ HC QAM-16 signal is shown in Fig. 9(i). The EVM curves show a very good matching with the SNR curves as we expected. Thus, it is evident that beam steering allocates the power peak to a specified location. The resulting SNR enhancement allows a good signal performance (in terms of EVM). Two representative constellations at a peak and a null are shown in Fig. 10(c) and (d). At the peak, the EVM is $7 \%$ and the constellations are well converged as shown in Fig. 10(c). Its RF spectrum is shown in Fig. 10(f). At the null, the received power is reduced by $14 \mathrm{~dB}$. Such low received power cannot even allow the demodulation of HC QAM-16 signal. Its RF spectrum shown in Fig. 10(g) indicates an evident power suppression.

\section{CONCLUSION}

In this paper, we explore the mm-wave beam steered fiberwireless systems for $5 \mathrm{G}$ indoor coverage, in terms of network architectures, key enabling devices and fiber-wireless links. First, we explore two kinds of indoor fiber-wireless network architectures for such mm-wave beam steering. Then we discuss and characterize the key enabling device of arrayed waveguide grating feedback loop (AWG-loop). Based on the AWG-loop, we further design two fiber-wireless links to accommodate the two network architectures. Both links with bit rates from $50 \mathrm{Mb} / \mathrm{s}$ to $8 \mathrm{~Gb} / \mathrm{s}$ per spatial channel are experimentally demonstrated with a $38-\mathrm{GHz}$ carrier frequency.

The advanced reversely modulated optical transmitter and half-cycled QAM-16 modulation are employed to realize a simplified mm-wave beam steered fiber-wireless link with a record-breaking 16 bits/s/Hz ( 4 spatial channels $\times 4$ bits $/ \mathrm{s} / \mathrm{Hz}$ ) spatial-spectral efficiency in its kind. The mm-wave beam steering is also observed. Four mm-wave beams with different directions are experimentally demonstrated with a $14-\mathrm{dBm}$ spatial isolation between each other.

\section{ACKNOWLEDGMENT}

Authors would like to thank Yanlu Li from Photonics Research Group, INTEC-department, Ghent University for his valuable technical discussion and proof reading.

\section{REFERENCES}

[1] Q. C. Li, H. Niu, A. T. Papathanassiou, and G. Wu, "5G network capacity: Key elements and technologies," IEEE Veh. Technol. Mag., vol. 9, no. 1, pp. 71-78, Mar. 2014.

[2] Y. Wang, J. Li, L. Huang, Y. Jing, A. Georgakopoulos, and P. Demestichas, "5G mobile: Spectrum broadening to higher-frequency bands to support high data rates," IEEE Veh. Technol. Mag., vol. 9, no. 3, pp. 39-46, Sep. 2014.

[3] W. Roh et al., "Millimeter-wave beamforming as an enabling technology for 5G cellular communications: Theoretical feasibility and prototype results," IEEE Commun. Mag., vol. 52, no. 2, pp. 106-113, Feb. 2014.

[4] T. Koonen and Z. Cao, "Optically controlled 2D radio beam steering system," in Proc. Int. Topical Meeting Microw. Photon. (MWP), 9th Asia-Pacific Microw. Photon. Conf. (APMP), 2014, pp. 389-391.

[5] Z. Cao et al., "Cyclic additional optical true time delay for microwave beam steering with spectral filtering," Opt. Lett., vol. 39, pp. 3402-3405, Jun. 2014.

[6] Z. Cao et al., "Spatial filtering in a broadband in-home OFDM radioover-fiber network," IEEE Photon. Technol. Lett., vol. 26, no. 6, pp. 575-578, Mar. 15, 2014.

[7] R. Bonjour, S. A. Gebrewold, D. Hillerkuss, C. Hafner, and J. Leuthold, "Ultra-fast tunable true-time delay using complementary phase-shifted spectra (CPSS)," in Proc. Opt. Fiber Commun. Conf. Exhibit. (OFC), 2015, pp. 1-3.

[8] L. Yaron, R. Rotman, S. Zach, and M. Tur, "Photonic beamformer receiver with multiple beam capabilities," IEEE Photon. Technol. Lett., vol. 22, no. 23, pp. 1723-1725, Dec. 1, 2010.

[9] O. Raz, S. Barzilay, R. Rotman, and M. Tur, "Submicrosecond scanangle switching photonic beamformer with flat RF response in the C and X bands," J. Lightw. Technol., vol. 26, no. 15, pp. 2774-2781, Aug. 1, 2008.

[10] B. M. Jung, J. D. Shin, and B. G. Kim, "Optical true time-delay for two-dimensional $X$-band phased array antennas," IEEE Photon. Technol. Lett., vol. 19, no. 12, pp. 877-879, Jun. 15, 2007.

[11] B. Vidal, T. Mengual, C. Ibanez-Lopez, and J. Marti, "Optical beamforming network based on fiber-optical delay lines and spatial light modulators for large antenna arrays," IEEE Photon. Technol. Lett., vol. 18, no. 24, pp. 2590-2592, Dec. 15, 2006.

[12] Z. Cao et al., "Advanced integration techniques on broadband millimeter-wave beam steering for $5 \mathrm{G}$ wireless networks and beyond," IEEE J. Quantum Electron., vol. 52, no. 1, Jan. 2016, Art. no. 0600620.

[13] R. Bonjour et al., "Ultra-fast millimeter wave beam steering," IEEE $J$. Quantum Electron., vol. 52, no. 1, Jan. 2016, Art. no. 0600708.

[14] M. A. Piqueras et al., "Optically beamformed beam-switched adaptive antennas for fixed and mobile broad-band wireless access networks," IEEE Trans. Microw. Theory Techn., vol. 54, no. 2, pp. 887-899, Feb. 2006.

[15] M. S. Rasras et al., "Integrated resonance-enhanced variable optical delay lines," IEEE Photon. Technol. Lett., vol. 17, no. 4, pp. 834-836, Apr. 2005

[16] L. Zhuang et al., "Novel ring resonator-based integrated photonic beamformer for broadband phased array receive antennas-Part II: Experimental prototype," J. Lightw. Technol., vol. 28, no. 1, pp. 19-31, Jan. $1,2010$.

[17] T. P. McKenna, J. A. Nanzer, and T. R. Clark, "Photonic beamsteering of a millimeter-wave array with 10-Gb/s data transmission," IEEE Photon. Technol. Lett., vol. 26, no. 14, pp. 1407-1410, Jul. 15, 2014.

[18] Z. Cao et al., "Integrated remotely tunable optical delay line for millimeter-wave beam steering fabricated in an InP generic foundry," Opt. Lett., vol. 40, no. 17, pp. 3930-3933, Sep. 2015.

[19] Z. Cao, Q. Wang, N. Tessema, X. Leijtens, F. Soares, and T. Koonen, "Beam steered millimeter-wave fiber-wireless system for $5 \mathrm{G}$ indoor coverage," in Proc. Opt. Fiber Commun. Conf., 2016, p. TU2B.1.

[20] N. M. Tessema, F. Yan, Z. Cao, E. Tangdiongga, and A. M. J. Koonen, "Compact and tunable AWG-based true-Time delay for multi-Gbps radio beamformer," in Proc. Eur. Conf. Opt. Commun. (ECOC), 2015, pp. 1-3. 


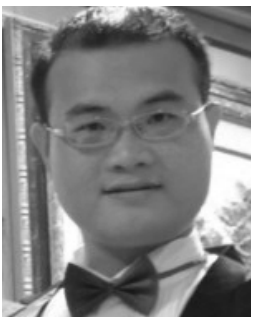

Z. Cao (M'15) received the B.Sc. degree in electronic information science and technology from Hunan Normal University, Changsha, China, the M.Eng. degree in telecom engineering from Hunan University, Changsha, and the Ph.D. (cum laude) degree (Hons.) from the Eindhoven University of Technology, Eindhoven, The Netherlands. His $\mathrm{Ph} . \mathrm{D}$. research was funded by the NWO project "SOWICI," mainly focusing on energy efficient access/indoor optical networks empowered by integrated optics, low-complexity digital signal processing, and flexible optical network design, under the supervision of Prof. Ton Koonen. The research activities produce a series of interesting scientific results. He has published 15 first-author peer-reviewed IEEE/OSA journal articles, including an invited paper in IEEE JLT and an invited review in IEEE JQE. He holds two granted Chinese patents and one US provisional patent. His research interests include modeling and design of integrated photonics circuits, microwave photonics, advanced DSP, and physical layer design of optical network. He was a recipient of Graduate Student Fellowship of the IEEE Photonics Society 2014. He was awarded the Outstanding thesis of the master's degree of Hunan Province, in 2010. He serves as an Active Reviewer for many top IEEE/OSA journals. He also has an invited talk about integrated optical radio beam steering systems in PIERS2014 and an invited talk about RF-OAM in ACP2015. Currently, his research articles have been cited for 749 times, with $\mathrm{H}$-index of 15 and 'i10' factor of 23 (source from google scholar).

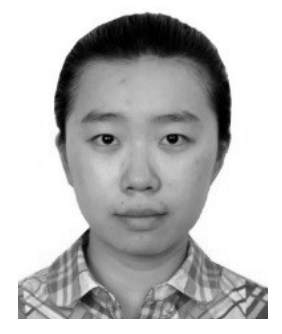

X. Zhao received the B.S. degree in information engineering from the Beijing Institute of Technology, Beijing, China in 2011, and the M.S. degree in electrical engineering from the Eindhoven University of Technology, Eindhoven, The Netherlands, in 2015.

She is currently pursuing the Ph.D. degree with the Electro-Optical Communications Group, Eindhoven University of Technology, where she is involved in the design and fabrication of integrated receivers for free space optical communication systems.

F. M. Soares received the M.Sc. degree in electrical engineering from the Delft University of Technology, The Netherlands, in 2000, and the Ph.D. degree in 2006 from the Eindhoven University of Technology. He was a PostDoctoral Researcher with the University of California at Davis, U.S.A., for four years, where he continued his research on the design, fabrication, and characterization of InP-based photonic integrated circuits. He joined the Photonic Components Department, Fraunhofer Institute for Telecommunications, Heinrich Hertz Institute, in 2010, where he has been involved in the framework of various EU-funded Projects, such as EuroPIC, PARADIGM, ACTPHAST, and PhoxTrot. His interests lie in the large-scale-, and high-level- integration of InP-based photonic devices.

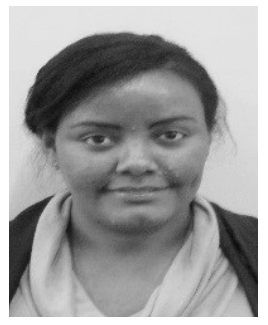

N. Tessema received the B.Sc. degree in electrical engineering from Mekelle University, Mekelle, Ethiopia, in 2007, and the master's degree in telecommunications engineering from the Delft university of Technology, Delft, The Netherlands, in 2012. She was with Ethio Telcom for the next three years. She is currently pursuing the Ph.D. degree with the Electro-Optic Communications Group, Eindhoven University of Technology, Eindhoven, The Netherlands.

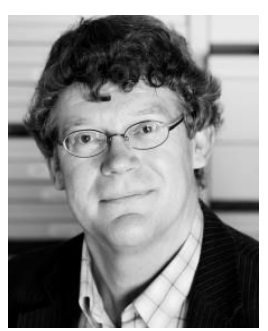

A. M. J Koonen (F'07) received the (cum laude) degree in electrical engineering from the Eindhoven University of Technology (TU/e) in 1979. He was involved in industrial research over 20 years with Philips' Telecommunicatie Industrie, AT\&T Network Systems, and Lucent Bell Laboratories on high-speed transmission systems and optical fiber systems for hybrid access networks, from 1987, as a Technical Manager. He was a part-time Professor with Twente University from 1991 to 2000. Since 2001, he has been a full-time Professor with TU/e. Since 2004, he has been the Chairman of the Electro-Optical Communication Systems Group, Department of Electrical Engineering, part of the COBRA Institute. Since 2012, he has been the Vice-Dean of the Department of Electrical Engineering, TU/e, where he was appointed to overlook EE's research activities. In 2014, he was a Distinguished Guest Professor with Hunan University, Changsha, China. He participated in many projects funded by the European Commission (EC), from the RACE program up to the FP7 program. He initiated and led projects in dynamically reconfigurable optical access networks, fiber-wireless networks, and label-controlled optical packet-switched networks in the FP4-FP6 programs. In FP7, he led/leads activities in the projects ALPHA, BONE, POF-PLUS, and MODE-GAP. $\mathrm{He}$ also started and led a number of nationally funded projects in these fields. $\mathrm{He}$ is a frequent Reviewer of EC projects. He was the Chairman of Panel PE7A for the European Research Council's Advanced Grant program, and a member of several Dutch Research and Development program committees. His current research interests are in access and in-building fiber network techniques, including multimode fiber networks and radio-over-fiber systems, advanced optical multiplexing schemes (e.g., mode multiplexing), and optical wireless communication. He has (co-)authored over 650 papers on optical fiber communication. He holds five U.S. patents ( +1 pending), and 1 Dutch patent. He is a fellow of Lucent Bell Labs in 1998 and OSA in 2013. He is also a 2011 recipient of an Advanced Investigator Grant of the European Research Council. 